OS2 (3) - 11 (OS02W0120)

\title{
Change of Ultrasonic Attenuation and Microstructure Evolution During Creep of Nickel Base Superalloy
}

\author{
Toshihiro Ohtani, Ndc Project, Ebara Research Co., Ltd., Kanagawa, Japan \\ Hirotsugu Ogi, Dept. of Engineering Science, Osaka Univ., Osaka, Japan \\ Masahiko Hirao, Dept. of Engineering Science, Osaka Univ., Osaka, Japan
}

We studied the microstructure evolution of a Nickel base superalloy (WASPALOY) during creep by monitoring ultrasonic attenuation. We applied tensile stress, ranging between 140 and $160 \mathrm{MPa}$, to the material at $1073 \mathrm{~K}$ and measured ultrasonic attenuation using the electromagnetic acoustic resonance (EMAR) technique. EMAR is a combined technique of the resonant acoustic technique and a non-contacting electromagnetic acoustic transducer (EMAT). Incorporation of EMAT in a resonant measurement contributes to improve the weak coupling efficiency to a large extent. The attenuation measurement is inherently free from any energy loss, resulting in the pure attenuation in a metal sample. Conventional contact transducers usually cause large loss, which hinders measuring attenuation in metals. We used a bulk-wave EMAT for transmitting and receiving the polarized shear wave traveling in the thickness direction of the sample to obtain the ultrasonic velocity from the resonant frequency and then the attenuation coefficient from the ringdown curve at resonance.

Two different creep tests were conducted; interrupted and continuous tests. In the interrupted test, we stopped creep loading and furnace-cooled the samples. After measuring the attenuation coefficients, we restarted the creep test. We repeated this procedure for every 20 or 100 hours until the rupture. In continuous test, we kept the loading until the creep strain reached a target value. We thus obtained a series of crept samples with various strains and measured their attenuation coefficients. To compare these two tests, we estimated the rupture time, tr, with the modified $\theta$ function and a rupture parameter, $\mathrm{P} \alpha$, and we calculated the estimated life fraction, $t / t \mathrm{t}$, from the relationship between creep strain and elapsed time. As a result, a good relationship between the attenuation coefficient and $t / t r$ was obtained. Furthermore, we observed the microstructure evolution with optical, SEM and TEM microscopes. The change of the attenuation coefficient corresponded to the evolution of the dislocation structure. The relationship between the attenuation coefficient and the dislocation structure can be explained by the string model of dislocation vibration. EMAR has a potential to assess the damage advance and to predict the remaining creep life of metals. 